\title{
Superposition solutions to the extended KdV equation for water surface waves
}

\author{
Piotr Rozmej@ • Anna Karczewska • Eryk Infeld
}

Received: 22 April 2017 / Accepted: 6 November 2017 / Published online: 16 November 2017

(C) The Author(s) 2017. This article is an open access publication

\begin{abstract}
The $\mathrm{KdV}$ equation can be derived in the shallow water limit of the Euler equations. Over the last few decades, this equation has been extended to include higher-order effects. Although this equation has only one conservation law, exact periodic and solitonic solutions exist. Khare and Saxena (Phys Lett A 377:27612765, 2013; J Math Phys 55:032701, 2014; J Math Phys $56: 032104,2015)$ demonstrated the possibility of generating new exact solutions by combining known ones for several fundamental equations (e.g., Korteweg-de Vries, nonlinear Schrödinger). Here we find that this construction can be repeated for higher-order, nonintegrable extensions of these equations. Contrary to many statements in the literature, there seems to be no correlation between integrability and the number of nonlinear one variable wave solutions.
\end{abstract}

Keywords Shallow water waves - Extended KdV equation $\cdot$ Analytic solutions $\cdot$ Nonlinear equations

P. Rozmej $(\varangle)$

Faculty of Physics and Astronomy, University of Zielona

Góra, Szafrana 4a, 65-246 Zielona Góra, Poland

e-mail: P.Rozmej@if.uz.zgora.pl

A. Karczewska

Faculty of Mathematics, Computer Science and

Econometrics, University of Zielona Góra, Szafrana 4a,

65-246 Zielona Góra, Poland

e-mail: A.Karczewska@wmie.uz.zgora.pl

E. Infeld

National Centre for Nuclear Research, Hoża 69,

00-681 Warszawa, Poland

e-mail: Eryk.Infeld@ncbj.gov.pl

\section{Introduction}

A long time ago, Stokes opened the field of nonlinear hydrodynamics by showing that waves described by nonlinear models can be periodic [1]. Although several related results followed, it took half a century before the Korteweg-de Vries equation became widely known [2]. A more accurate equation system, Boussinesq, was formulated in 1871. It is also the theme of several recent papers $[3,4]$. Another direction research has gone in is including perpendicular dynamics in $\mathrm{KdV}$, e.g., [5].

The $\mathrm{KdV}$ equation is one of the most successful physical equations. It consists of the mathematically simplest possible terms representing the interplay of nonlinearity and dispersion. This simplicity may be one of the reasons for success. Here we investigate this equation, improved as derived from the Euler inviscid and irrotational water equations.

Just as for conventional $\mathrm{KdV}$, two small parameters are assumed: wave amplitude/depth $(a / H)$ and depth/wavelength squared $(H / l)^{2}$. These dimensionless expansion constants are called $\alpha$ and $\beta$. We take the expansion one-order higher. The new terms will then be of second order. This procedure limits considerations to waves for which the two parameters are comparable. Unfortunately some authors tend to be careless about this limitation.

The next approximation to Euler's equations for long waves over a shallow riverbed is ( $\eta$ is the elevation above a flat surface divided by $H$ ) 


$$
\begin{aligned}
\eta_{t} & +\eta_{x}+\frac{3}{2} \alpha \eta \eta_{x}+\frac{1}{6} \beta \eta_{3 x}-\frac{3}{8} \alpha^{2} \eta^{2} \eta_{x} \\
& +\alpha \beta\left(\frac{23}{24} \eta_{x} \eta_{2 x}+\frac{5}{12} \eta \eta_{3 x}\right)+\frac{19}{360} \beta^{2} \eta_{5 x}=0
\end{aligned}
$$

In (1) subsequently we use low indexes for derivatives $\left(\eta_{n x} \equiv \frac{\partial^{n} \eta}{\partial x^{n}}\right)$. This second-order equation was called by Marchant and Smyth $[6,7]$ the extended $K d V$. It was also derived in a different way in [8] and $[9,10]$. We call it $K d V 2$. It is not integrable. However, by keeping the same terms but changing one numerical coefficient (specifically, replacing $\frac{23}{24}$ by $\frac{5}{6}$ ) we can obtain an integrable equation $[11,12]$.

Not only is KdV2 non-integrable, it only seems to have one conservation law (volume or mass) [13]. However, a simple derivation of adiabatically conserved quantities can be found in [14].

Recently, Khare and Saxena [15-17] demonstrated that for several nonlinear equations which admit solutions in terms of elliptic functions $\mathrm{cn}(x, m), \mathrm{dn}(x, m)$ there exist solutions in terms of superpositions $\mathrm{cn}(x, m)$ $\pm \sqrt{m} \mathrm{dn}(x, m)$. They also showed that $\mathrm{KdV}$ which admits solutions in terms of $\mathrm{dn}^{2}(x, m)$ also admits solutions in terms of superpositions $\mathrm{dn}^{2}(x, m) \pm$ $\sqrt{m} \mathrm{cn}(x, m) \mathrm{dn}(x, m)$. Since then we found analytic solutions to $\operatorname{KdV} 2$ in terms of $\mathrm{cn}^{2}(x, m)[18,19]$, the results of Khare and Saxena [15-17] inspired us to look for solutions to KdV2 in similar form.

\section{Exact periodic solutions for $\mathrm{KdV} 2$}

First, we repeat shortly the results obtained by Khare and Saxena [15], but formulating them for $\mathrm{KdV}$ in a fixed frame, that is, for the equation

$\eta_{t}+\eta_{x}+\frac{3}{2} \alpha \eta \eta_{x}+\frac{1}{6} \beta \eta_{3 x}=0$.

Assuming solution in the form

$$
\eta(x, t)=A \mathrm{dn}^{2}[B(x-v t), m]
$$

one finds

$$
\begin{aligned}
A= & \frac{4}{3} \frac{B^{2} \beta}{\alpha} \text { and } v=1+\frac{2}{3} \beta B^{2}(2-m) \\
& =1+\frac{\alpha}{2} A(2-m) .
\end{aligned}
$$

Next, the authors [15] showed that superpositions

$$
\begin{aligned}
\eta_{ \pm}(x, t)= & \frac{1}{2} A\left(\operatorname{dn}^{2}[B(x-v t), m]\right. \\
& \pm \sqrt{m} \operatorname{cn}[B(x-v t), m] \operatorname{dn}[B(x-v t), m])
\end{aligned}
$$

are solutions to (2) with the same relation between $A$ and $B$, but for a different velocity, $v_{ \pm}=1+\frac{1}{6} \beta B^{2}(5-m)$.

Now, we look for periodic nonlinear wave solutions of KdV2 (1). Introduce $y:=x-v t$. Then $\eta(x, t)=$ $\eta(y), \eta_{t}=-v \eta_{y}$ and Eq. (1) takes the form of an ODE

$$
\begin{aligned}
& (1-v) \eta_{y}+\frac{3}{2} \alpha \eta \eta_{y}+\frac{1}{6} \beta \eta_{3 y}-\frac{3}{8} \alpha^{2} \eta^{2} \eta_{y} \\
& +\alpha \beta\left(\frac{23}{24} \eta_{y} \eta_{2 y}+\frac{5}{12} \eta \eta_{3 y}\right) \\
& \quad+\frac{19}{360} \beta^{2} \eta_{5 y}=0 .
\end{aligned}
$$

\subsection{Single periodic function $\mathrm{dn}^{2}$}

First, we recall some properties of the Jacobi elliptic functions (arguments are omitted)

$$
\mathrm{sn}^{2}+\mathrm{cn}^{2}=1, \quad \mathrm{dn}^{2}+m \mathrm{sn}^{2}=1 .
$$

Their derivatives are

$$
\begin{aligned}
& \frac{\mathrm{d} s \mathrm{~s}}{\mathrm{~d} y}=\mathrm{cn} \mathrm{dn}, \quad \frac{\mathrm{d} \mathrm{cn}}{\mathrm{d} y}=-\mathrm{sn} \mathrm{dn}, \\
& \frac{\mathrm{d} \mathrm{dn}}{\mathrm{d} y}=-m \text { sn cn. }
\end{aligned}
$$

Assume a solution of (1) in the same form as $\mathrm{KdV}$ solution (3). Insertion of (3) into (5) yields

$$
\frac{A B m}{180} \mathrm{cndn} \operatorname{sn}\left(F_{0}+F_{2} \mathrm{cn}^{2}+F_{4} \mathrm{cn}^{4}\right)=0 .
$$

Equation (8) holds for arbitrary arguments when $F_{0}, F_{2}, F_{4}$ vanish simultaneously. The explicit form of this set of equations is following

$$
\begin{aligned}
F_{0}= & 135 \alpha^{2} A^{2}(m-1)^{2}+30 \alpha A(m-1) \\
& \times\left(\beta B^{2}(63 m-20)+18\right) \\
& -8\left(19 \beta^{2} B^{4}\left(17 m^{2}-17 m+2\right)\right.
\end{aligned}
$$




$$
\begin{aligned}
& \left.+30 \beta B^{2}(2 m-1)+45\right) \\
& +360 v=0 \\
F_{2}= & -30 m\left[9 \alpha^{2} A^{2}(m-1)+6 \alpha A\right. \\
& \times\left(\beta B^{2}(32 m-21)+3\right)-8 \beta B^{2} \\
& \left.\times\left(19 \beta B^{2}(2 m-1)+3\right)\right]=0 \\
F_{4}= & 45 m^{2}\left(3 \alpha^{2} A^{2}+86 \alpha A \beta B^{2}-152 \beta^{2} B^{4}\right)=0 .
\end{aligned}
$$

Equation (11) is equivalent to the [10, Eq. (26)] obtained for solitonic solutions to KdV2. Denoting $z:=\frac{B^{2} \beta}{A \alpha}$ one obtains from (11) two possible solutions

$z_{1}=\frac{43-\sqrt{2305}}{152}<0$ and $z_{2}=\frac{43+\sqrt{2305}}{152}>0$.

The case $z=z_{1}$ leads to $B^{2}<0$ and has to be rejected as in previous papers $[10,18]$. Then for $z=z_{2}$ the amplitude $A$ is

$A=\frac{43+\sqrt{2305}}{3} \frac{B^{2} \beta}{\alpha}>0$.

Inserting this into (10) yields

$$
B^{2}=\frac{3(\sqrt{2305}-14)}{703 \beta(2-m)}
$$

and then using (13) one has

$$
A=\frac{3(\sqrt{2305}-51)}{37 \alpha(m-2)} .
$$

Finally from (9) one obtains

$$
\begin{aligned}
v= & \left\{4(129877+314 \sqrt{2305}) m^{2} .\right. \\
& +(18409 \sqrt{2305}-3209623)(m-1)\} \\
& /\left\{520220(m-2)^{2}\right\}
\end{aligned}
$$

Despite the same form of solutions to $\mathrm{KdV}$ and $\mathrm{KdV} 2$, there is a fundamental difference. $\mathrm{KdV}$ only

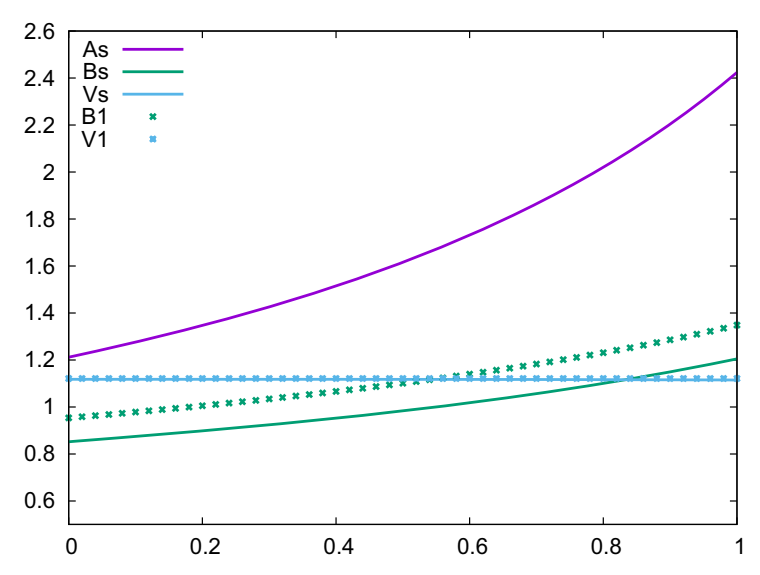

Fig. 1 Coefficients $A, B, v,\left(z=z_{2}\right)$ as functions of $m$ for the periodic solution of $\mathrm{KdV} 2$ in the form of a single function (3). Lines represent KdV2 coefficients, points KdV coefficients. $\left(A_{\mathrm{KdV}}=A_{\mathrm{KdV} 2}\right) .($ Color figure online $)$

imposes two conditions on coefficients $A, B, v$ for given $m$, so there is one parameter freedom. This is no longer the case for KdV2.

\subsection{Comparison to KdV solutions}

Is a solution of $\mathrm{KdV} 2$ much different from the $\mathrm{KdV}$ solution for the same $m$ ? In order to compare solutions of both equations, remember that the set of three Eqs. (9)-(11) fixes all $A, B, v$ coefficients for $\mathrm{KdV} 2$ for given $m$. In the case of $\mathrm{KdV}$, the equation analogous to (8) only imposes two conditions on three parameters. Therefore, one parameter, say amplitude $A$, can be chosen arbitrary. Then we compare coefficients of solutions to $\mathrm{KdV} 2$ and $\mathrm{KdV}$ choosing the same value of $A$, that is, $A_{\mathrm{KdV} 2}$. Such comparison is displayed in Fig. 1 for $\alpha=\beta=\frac{1}{10}$.

It is clear that $v_{\mathrm{KdV} 2}$ and $v_{\mathrm{KdV}}$ are very similar. We have the following relations: for $\mathrm{KdV} \frac{B^{2}}{A}=\frac{3 \alpha}{4 \beta}$, whereas for $\operatorname{KdV} 2 \frac{B^{2}}{A}=\frac{\alpha}{\beta} z_{2}$. Since $z_{2} \approx 0.6$, $B_{\mathrm{KdV}} / B_{\mathrm{KdV}}=\sqrt{\frac{3}{4 z_{2}}} \approx 1.12$. The same relations hold between $\mathrm{KdV} 2$ and $\mathrm{KdV}$ coefficients for superposition solutions shown in Fig. 3.

The above examples for the case $\alpha=\beta=\frac{1}{10}$ show that for somewhat small values of $\alpha$ the coefficients of $\mathrm{KdV} 2 \mathrm{dn}^{2}$ solutions are not much different from those of $\mathrm{KdV}$.

However, physically relevant exact solutions of $\mathrm{dn}^{2}$ to $\mathrm{KdV} 2$ can be found for much larger values of the 


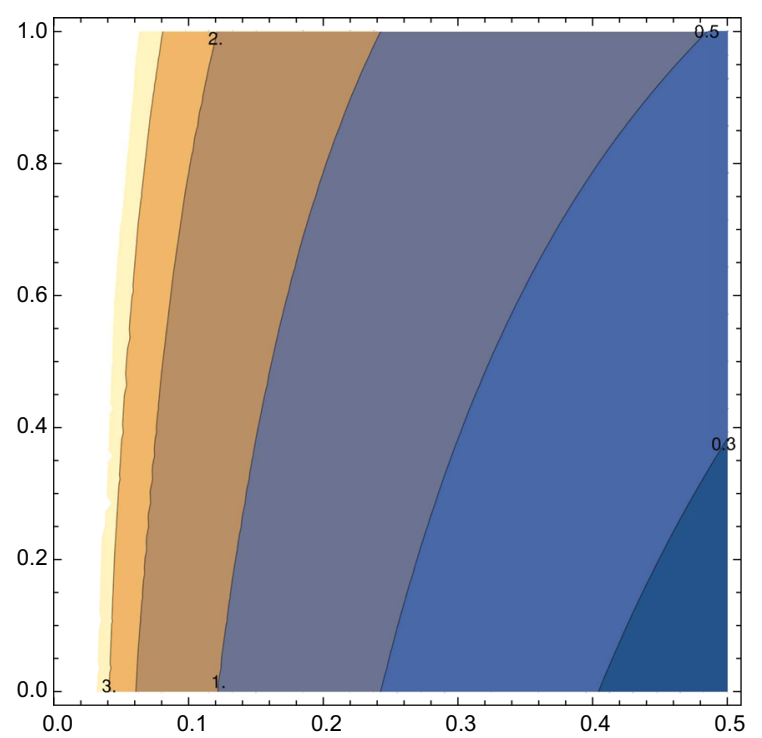

Fig. 2 Amplitude $A(\alpha, m)$ given by (15) as function of $(\alpha, m)$. (Color figure online)

parameter $\alpha$. In Fig. 2, the amplitude $A(\alpha, m)$ given by (15) is shown as a contour plot for the region $\alpha \in[0.01,0.5], m \in[0,1]$. It is clear that reasonable amplitudes occur in wide regions of $\alpha$ and $m$ values. The amplitudes become too big only for $\alpha \rightarrow 0$, but in such cases KdV works very well.

\subsection{Superposition " $\mathrm{dn}^{2}+\sqrt{m} \mathrm{cn} d \mathrm{dn}^{2}$}

Now assume the periodic solution to be in the same form as the corresponding superposition solution of $\mathrm{KdV}$ [15] function

$$
\begin{gathered}
\eta_{+}(y)=\frac{1}{2} A\left[\operatorname{dn}^{2}(B y, m)+\sqrt{m} \operatorname{cn}(B y, m)\right. \\
\operatorname{dn}(B y, m)],
\end{gathered}
$$

where $A, B, v$ are yet unknown constants. ( $m$ is the elliptic parameter.) We will need

$$
\begin{aligned}
\eta_{y}= & -\frac{1}{2} A B \sqrt{m}(\sqrt{m} \mathrm{cn}+\mathrm{dn})^{2} \mathrm{sn}, \\
\eta_{2 y}= & \frac{1}{2} A B^{2} \sqrt{m} \\
& \times(\sqrt{m} \mathrm{cn}+\mathrm{dn})^{2}\left(-\mathrm{cn} \mathrm{dn}+2 \sqrt{m} \mathrm{sn}^{2}\right), \\
\eta_{3 y}= & \frac{1}{2} A B^{3} \sqrt{m}(\sqrt{m} \mathrm{cn}+\mathrm{dn})^{2} \\
& \times \mathrm{sn}\left(m \mathrm{cn}^{2}+6 \sqrt{m} \mathrm{cndn}+\mathrm{dn}^{2}-4 m \mathrm{sn}^{2}\right), \\
\eta_{5 y}= & -\frac{1}{2} A B^{5} \sqrt{m}(\sqrt{m} \mathrm{cn}+\mathrm{dn})^{2}
\end{aligned}
$$

$$
\begin{aligned}
& \times \mathrm{sn}\left[m^{2} \mathrm{cn}^{4}+30 m^{3 / 2} \mathrm{cn}^{3} \mathrm{dn}+\mathrm{dn}^{4}-44 \mathrm{dn}^{2} \mathrm{sn}^{2}\right. \\
& +16 m^{2} \mathrm{sn}^{4}-30 \sqrt{m} \mathrm{cndn}\left(-\mathrm{dn}^{2}+4 m \mathrm{sn}^{2}\right) \\
& \left.+\mathrm{cn}^{2}\left(74 m \mathrm{dn}^{2}-44 m^{2} \mathrm{sn}^{2}\right)\right]
\end{aligned}
$$

Denote (5) as

$E_{1}+E_{2}+E_{3}+E_{4}+E_{5}+E_{6}+E_{7}=0$,

where

$$
\begin{aligned}
& E_{1}=(1-v) \eta_{y}=-\frac{1}{2} A B(1-v) \sqrt{m}(\sqrt{m} \mathrm{cn}+\mathrm{dn})^{2} \mathrm{sn}, \\
& E_{2}=\frac{3}{2} \alpha \eta \eta_{y}=-\frac{3}{8} \alpha A^{2} B \sqrt{m}(\sqrt{m} \mathrm{cn}+\mathrm{dn})^{3} \mathrm{sndn},
\end{aligned}
$$

$$
\begin{aligned}
E_{3}= & \frac{1}{6} \beta \eta 3 y=\frac{1}{12} \beta A B^{3} \sqrt{m}(\sqrt{m} \mathrm{cn}+\mathrm{dn})^{2} \mathrm{sn} \\
& \times\left(m \mathrm{cn}^{2}+6 \sqrt{m} \mathrm{cndn}+\mathrm{dn}^{2}-4 m \mathrm{sn}^{2}\right) \\
E_{4}= & -\frac{3}{8} \alpha^{2} \eta^{2} \eta_{y}=\frac{3}{64} \alpha^{2} A^{3} B \sqrt{m} \mathrm{dn}^{2}(\sqrt{m} \mathrm{cn}+\mathrm{dn})^{4} \mathrm{sn} \\
E_{5}= & \frac{23}{24} \alpha \beta \eta_{y} \eta_{2 y}=-\frac{23}{96} \alpha \beta A^{2} B^{3} m(\sqrt{m} \mathrm{cn}+\mathrm{dn})^{4} \\
& \times \mathrm{sn}\left(-\mathrm{cndn}+2 \sqrt{m} \mathrm{sn}^{2}\right), \\
E_{6}= & \frac{5}{12} \alpha \beta \eta \eta_{3 y}=\frac{5}{48} \alpha \beta A^{2} B^{3} \sqrt{m} \mathrm{dn}(\sqrt{m} \mathrm{cn}+\mathrm{dn})^{3} \\
& \times \mathrm{sn}\left(m \mathrm{cn}^{2}+6 \sqrt{m} \mathrm{cndn}^{2} \mathrm{dn} n^{2}-4 m \mathrm{sn}^{2}\right) \\
E_{7}= & \frac{19}{360} \beta^{2} \eta_{5 y}=-\frac{19}{720} \beta^{2} A B^{5} \sqrt{m}(\sqrt{m} \mathrm{cn}+\mathrm{dn})^{2} \\
& \times \mathrm{sn}^{2}\left[m^{2} \mathrm{cn}^{4}+30 m^{3 / 2} \mathrm{cn}^{3} \mathrm{dn}+\mathrm{dn}^{4}-44 \mathrm{dn} \mathrm{sn}^{2}\right. \\
& +16 m^{2} \mathrm{sn}^{4}-30 \sqrt{m} \mathrm{cndn}^{2}\left(-\mathrm{dn}^{2}+4 m \mathrm{sn}^{2}\right) \\
& \left.+\mathrm{cn}^{2}\left(74 m \mathrm{dn}^{2}-44 m^{2} \mathrm{sn}^{2}\right)\right] .
\end{aligned}
$$

Then (22) becomes

$$
\begin{gathered}
\frac{1}{2} A B \sqrt{m}(\sqrt{m} \mathrm{cn}+\mathrm{dn})^{2} \mathrm{sn} \times\left(F_{0}+F_{c d} \mathrm{cn} \mathrm{dn}\right. \\
\left.+F_{c^{2}} \mathrm{cn}^{2}+F_{c^{3} d} \mathrm{cn}^{3} \mathrm{dn}+F_{c^{4}} \mathrm{cn}^{4}\right)=0 .
\end{gathered}
$$

Equation (30) is valid for arbitrary arguments when all coefficients $F_{0}, F_{c d}, F_{c^{2}}, F_{c^{3} d}, F_{c^{4}}$ vanish simultaneously. This gives us a set of equations for the coefficients $A, B, v$ 


$$
\begin{aligned}
F_{0}= & -1440 v-135 \alpha^{2} A^{2}(m-1)^{2} \\
& -60 \alpha A(m-1)\left[\beta B^{2}(48 m-5)+18\right] \\
& +4\left[19 \beta^{2} B^{4}\left(61 m^{2}-46 m+1\right)\right. \\
& \left.+60 \beta B^{2}(5 m-1)+360\right]=0 \\
F_{c d}= & 30 \sqrt{m}\left[9 \alpha^{2} A^{2}(m-1)\right. \\
& +3 \alpha A\left(\beta B^{2}(75 m-31)+12\right) \\
& \left.-4 \beta B^{2}\left(19 \beta B^{2}(5 m-1)+12\right)\right]=0 \\
F_{c^{2}}= & 15 m\left(27 \alpha^{2} A^{2}(m-1)\right. \\
& +12 \alpha A\left(\beta B^{2}(59 m-37)+6\right) \\
& \left.-32 \beta B^{2}\left(19 \beta B^{2}(2 m-1)+3\right)\right)=0 \\
F_{c^{3} d}= & -90 m^{3 / 2}\left(3 \alpha^{2} A^{2}+86 \alpha A \beta B^{2}\right. \\
& \left.-152 \beta^{2} B^{4}\right)=0, \\
F_{c^{4}}= & -90 m^{2}\left(3 \alpha^{2} A^{2}+86 \alpha A \beta B^{2}\right. \\
& \left.-152 \beta^{2} B^{4}\right)=0 .
\end{aligned}
$$

Equations (34) and (35) are equivalent and give the same condition as (11). Solving (34) with respect to $B^{2}$, we obtain the same relations as in [10, Eq. (28)]

$\left(B_{1 / 2}\right)^{2}=\frac{A \alpha}{\beta}\left(\frac{43 \mp \sqrt{2305}}{152}\right)$

Denote

$z_{1}=\frac{43-\sqrt{2305}}{152} \quad$ and $\quad z_{2}=\frac{43+\sqrt{2305}}{152}$.

It is clear that $z_{1}<0$ and $z_{2}>0 . B$ has to be realvalued. This is possible for the case $z=z_{1}$ if $A<$ 0 , and for $z=z_{2}$ if $A>0$. The value of $z_{2}$ is the same as that found for the exact soliton solution in [10, Eq. (28)]. In general

$B^{2}=\frac{A \alpha}{\beta} z$

Now, we insert (38) into (31),(32) and (33). Besides a trivial solution with $A=0$, we obtain

$$
\begin{aligned}
& 1440(1-v)+A \alpha(1-m)[1080-135 A \alpha(1-m)] \\
& \quad-240 A \alpha(1-5 m)-30(A \alpha)^{2}\left(10-109 m+96 m^{2}\right) z \\
& \quad+4(A \alpha)^{2}\left(19-847 m+1159 m^{2}\right) z^{2}=0, \\
& 9[A \alpha(m-1)+4]+3[A \alpha(75 m-31)-16] z \\
& \quad-76 A \alpha(5 m-1) z^{2}=0, \\
& 9(3 A \alpha(m-1)+8)+12(A \alpha(59 m-37)-8) z \\
& \quad-608 A \alpha(2 m-1) z^{2}=0 .
\end{aligned}
$$

From (40) we find

$$
A=-\frac{12(4 z-3)}{\alpha\left[76 z^{2}(5 m-1)-z(225 m-93)-9(m-1)\right]}
$$

but from (41) it follows that

$$
A=-\frac{24(4 z-3)}{\alpha\left[608 z^{2}(2 m-1)-4 z(177 m-111)-27(m-1)\right]}
$$

This looks like a contradiction, but substitution $z=$ $z_{1}=(43-\sqrt{2305}) / 152$ in both (42) and (43) gives the same result

$$
A_{1}=\frac{24(71+\sqrt{2305})}{(-329+5 \sqrt{2305}) \alpha(m-5)} .
$$

For $z=z_{2}=(43+\sqrt{2305}) / 152$, the common result is

$$
A_{2}=\frac{24(-71+\sqrt{2305})}{(329+5 \sqrt{2305}) \alpha(m-5)} .
$$

This means that not only are Eqs. (34) and (35) equivalent, but also (32) and (33), as well. Therefore, Eqs. (32)-(35) supply only three independent conditions for the coefficients of KdV2 solutions in the form (17).

Now, using $z=z_{1}$ and $A_{1}$ given by (44) we obtain from (39)

$v_{1}=\frac{\operatorname{vnum}_{-}(m)}{\operatorname{vden}_{-}(m)}$

and with $z=z_{2}$ and $A_{2}$ given by (45)

$$
v_{2}=\frac{\operatorname{vnum}_{+}(m)}{\operatorname{vden}_{+}(m)},
$$


where

$$
\begin{aligned}
\operatorname{vnum}_{\mp}(m)= & 6\left\{(2912513 \mp 58361 \sqrt{2305}) m^{2}\right. \\
& -54(584397 \mp 10069 \sqrt{2305}) m \\
& +75245133 \mp 1419141 \sqrt{2305}\},
\end{aligned}
$$

and

$\operatorname{vden}_{\mp}(m)=95(329 \mp 5 \sqrt{2305})^{2}(m-5)^{2}$.

\subsection{Discussion of mathematical solutions}

From a strictly mathematical point of view, we found two families of solutions determined by coefficients $A, B, v$ as functions of the elliptic parameter $m$. There are two cases.

Case $1 z=z_{1}=\frac{43-\sqrt{2305}}{152} \approx-0.0329633<0$.

This case leads to $B^{2}<0$ and has to be rejected as in previous papers $[10,18]$.

Case $2 z=z_{2}=\frac{43+\sqrt{2305}}{152} \approx 0.598753>0$. Then

$$
\begin{aligned}
& A=\frac{12(\sqrt{2305}-51)}{37 \alpha(m-5)}>0, \\
& B=\sqrt{\frac{12(\sqrt{2305}-14)}{703(5-m) \beta}}
\end{aligned}
$$

and $v_{2}$ is given by (47). Since $m \in[0,1],(m-5)<0$ then $B_{2}$ is real. The solution in this case is

$$
\begin{aligned}
& \eta_{2}\left(x-v_{2} t, m\right)=\frac{1}{2} A_{2}\left[\operatorname{dn}^{2}\left(B_{2}\left(x-v_{2} t\right), m\right)\right. \\
& \left.\quad+\sqrt{m} \operatorname{cn}\left(B_{2}\left(x-v_{2} t\right), m\right) \operatorname{dn}\left(B_{2}\left(x-v_{2} t\right), m\right)\right] .
\end{aligned}
$$

Coefficients $A_{2}, B_{2}, v_{2}$ of superposition solutions (17) to KdV2 as functions of $m$ are presented in Fig. 3 for $\alpha=\beta=\frac{1}{10}$ and compared to corresponding solutions to KdV. Here, similarly as in Fig. 1, we assume that $A_{\mathrm{KdV}}=A_{\mathrm{KdV} 2}$.

Physically relevant exact superposition solutions to $\mathrm{KdV} 2$ can be found for greater values of the parameter $\alpha$ than $\frac{1}{10}$. In Fig. 4 , the amplitude $A(\alpha, m)$ given by (48) is shown as a contour plot for the region

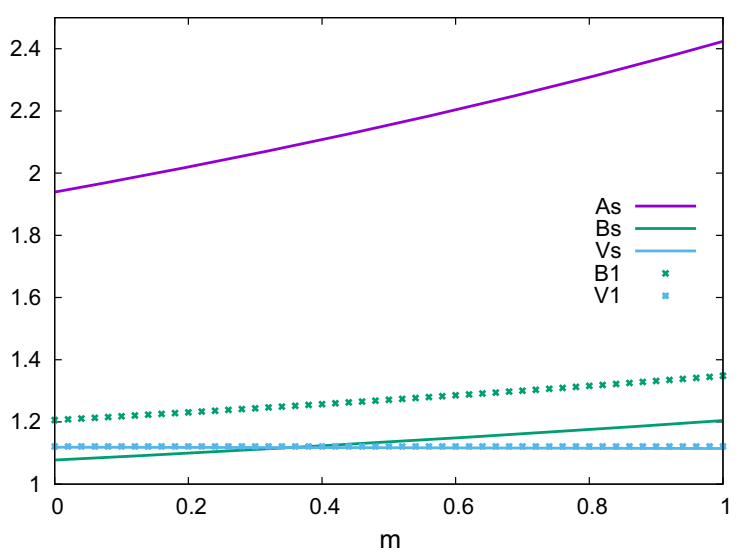

Fig. 3 Same as in Fig. 1 but for superposition solutions $\eta_{+}$(17) and $\eta_{-}$(51). (Color figure online)

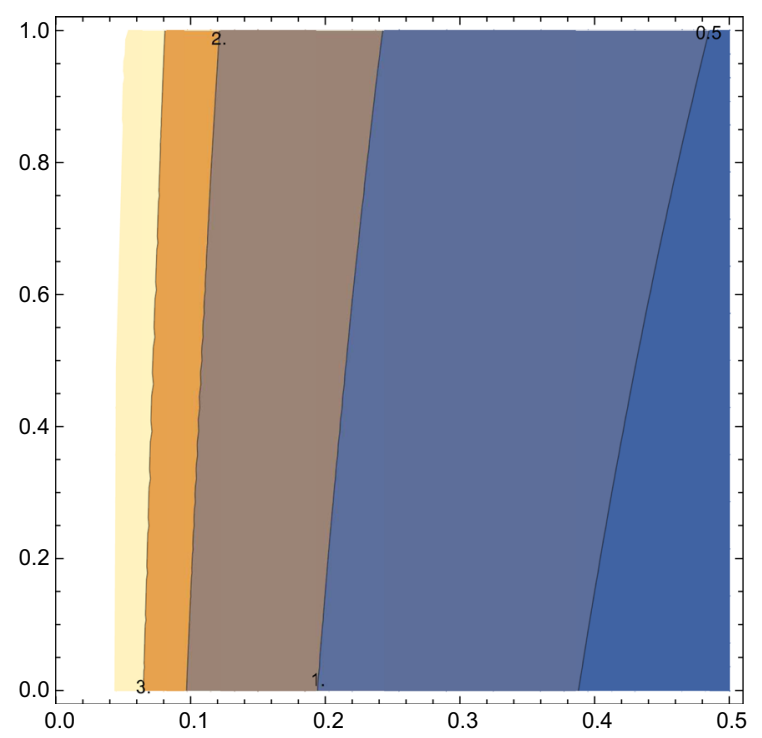

Fig. 4 Same as in Fig. 2 but for superposition solutions $\eta_{+}$. (Color figure online)

$\alpha \in[0.01,0.5], m \in[0,1]$. It is clear that reasonable amplitudes occur in wide regions of $\alpha$ and $m$ values, similarly like in the case $\mathrm{dn}^{2}$. The amplitudes become too big only for $\alpha \rightarrow 0$, but in such cases $\mathrm{KdV}$ works very well.

\subsection{Superposition 1 " $d n^{2}-\sqrt{m} \mathrm{cn} d \mathrm{dn}^{2}$}

Now we check the alternative superposition " $\mathrm{dn}^{2}-$ $\sqrt{m} \mathrm{cndn"}$ 
$\eta_{-}(y)=\frac{1}{2} A\left[\mathrm{dn}^{2}(B y, m)-\sqrt{m} \operatorname{cn}(B y, m) \operatorname{dn}(B y, m)\right]$.

In this case, the derivatives are given by formulas similar to (18)-(21) with some signs altered. Analogous changes occur in formulas (23)-(29). Then (22) has a similar form like (30)

$$
\begin{aligned}
& \frac{1}{2} A B \sqrt{m}(-\sqrt{m} \mathrm{cn}+\mathrm{dn})^{2} \mathrm{sn} \times\left(F_{0}+F_{c d} \mathrm{cndn}\right. \\
& \left.\quad+F_{c^{2}} \mathrm{cn}^{2}+F_{c^{3} d} \mathrm{cn}^{3} \mathrm{dn}+F_{c^{4}} \mathrm{Cn}^{4}\right)=0
\end{aligned}
$$

Equation (52) is valid for arbitrary arguments when all coefficients $F_{0}, F_{c d}, F_{c^{2}}, F_{c^{3} d}, F_{c^{4}}$ vanish simultaneously. This gives us a set of equations for the coefficients $v, A, B$. Despite some changes in signs on the way to (52), this set is the same as for " $\mathrm{dn}^{2}+$ $\sqrt{m}$ cn dn" superposition (31)-(35). Then the coefficients $A, B, v$ for superposition " $\mathrm{dn}^{2}-\sqrt{m}$ cn $\mathrm{dn}$ " are the same as for superposition " $\mathrm{dn}^{2}+\sqrt{m} \mathrm{cn} d \mathrm{n}$ " given above. This property for KdV2 is the same as for $\mathrm{KdV}$, see [15]. It follows from periodicity of the Jacobi elliptic functions. From

$$
\begin{aligned}
& \operatorname{cn}(y+2 K(m), m)=-\operatorname{cn}(y, m), \\
& \operatorname{dn}(y+2 K(m), m)=\operatorname{dn}(y, m)
\end{aligned}
$$

it follows that

$$
\begin{aligned}
& \mathrm{dn}^{2}(y+2 K(m), m) \\
& +\sqrt{(m)} \operatorname{cn}(x+2 K(m), m) \mathrm{dn}(x+2 K(m), m) \\
& =\mathrm{dn}^{2}(x, m)-\sqrt{(m)} \mathrm{cn}(x, m) \mathrm{dn}(x, m) .
\end{aligned}
$$

So both superpositions $\eta_{+}$(17) and $\eta_{-}$(51) represent the same solution, but shifted by the period of the Jacobi elliptic functions. This property is well seen in Figs. 5, 6 and 7.

\section{Examples}

Below, some examples of wave profiles for both $\mathrm{KdV}$ and $\mathrm{KdV} 2$ are presented. We know from Sect. 2 that for a given $m$, the coefficients $A, B, v$ of $\mathrm{KdV} 2$ solutions are fixed. As we have already written, this is not the case for $A, B, v$ of $\mathrm{KdV}$ solutions. So, there is one free parameter. In order to compare $\mathrm{KdV} 2$ solutions to those of $\mathrm{KdV}$ for identical $m$, we set $A_{\mathrm{KdV}}=A_{\mathrm{KdV} 2}$. In Figs. 5, 6 and 7 below, KdV solutions of the forms (3),

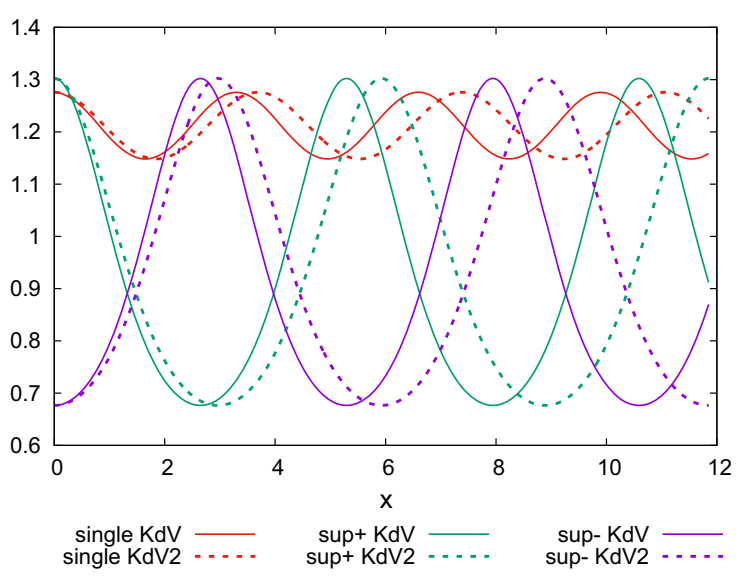

Fig. 5 Profiles of $\mathrm{KdV}$ and $\mathrm{KdV} 2$ waves for $m=0.1$. (Color figure online)

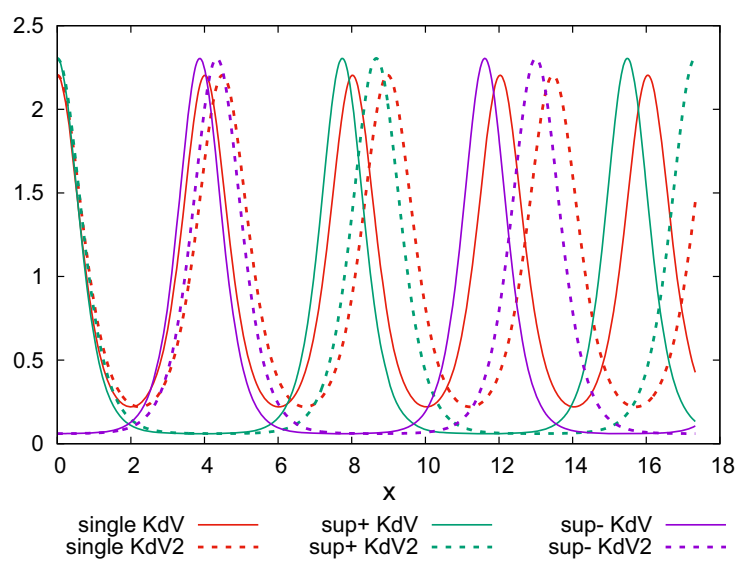

Fig. 6 Profiles of KdV and KdV2 waves for $m=0.9$. (Color figure online)

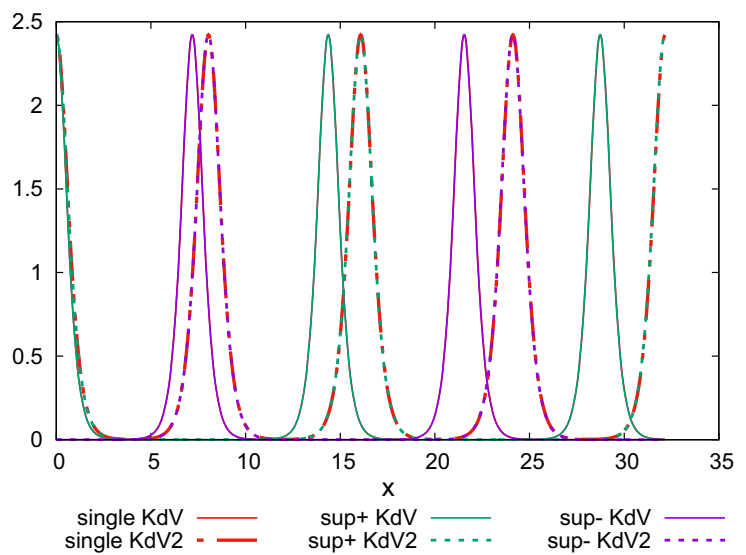

Fig. 7 Profiles of KdV and KdV2 waves for $m=0.99$. (Color figure online) 


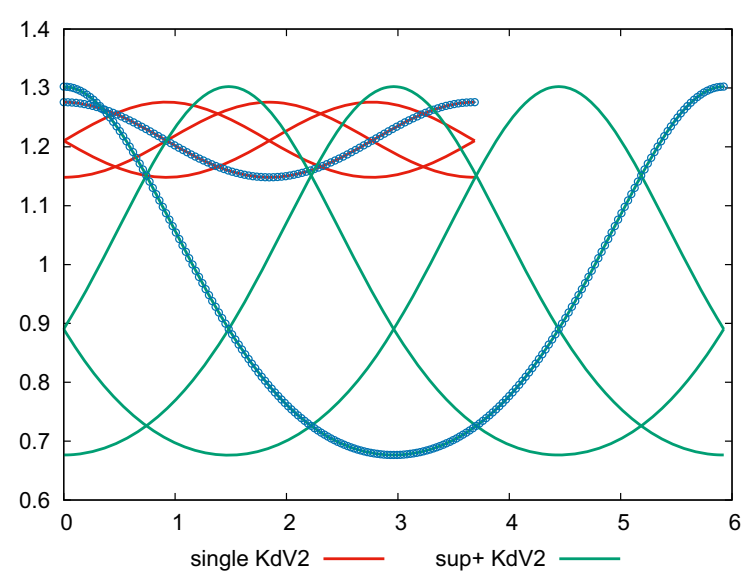

Fig. 8 Time evolution of single KdV2 solution (red lines) and $\eta_{+} \mathrm{KdV} 2$ solution for $m=0.1$. (Color figure online)

(17) and (51) are drawn with solid red, green and blue lines, respectively. For KdV2 solutions, the same color convention is used, but with dashed lines. In all the presented cases, the parameters $\alpha=\beta=0.1$ were used.

Comparison of wave profiles for different $m$ suggests several observations. For small $m$, solutions given by the single formula (3) differ substantially from those given by superpositions (17) and (51). Note that (3) is equal to the sum of both superpositions and when $m \rightarrow 1$ the distance between crests of $\eta_{+}$and $\eta_{-}$ increases to infinity (in the $m=1$ limit). All three solutions converge to the same soliton.

In order to check whether the obtained analytic solutions are really true solutions to KdV2 several numerical simulations were performed. In each of them, the numerical FDM code used with success in previous studies $[9-11,14,18,19]$ was applied. Since the calculations concerned periodic solutions, the periodic boundary conditions were used with an $x$ interval equal to the particular wavelength. In Figs. 5, 6 and 7 dashed lines display profiles of single $\mathrm{dn}^{2}(3)$ and superposition $\eta_{+}$ and $\eta_{-}$(4) solutions for three values of $m=0.1,0.9$ and 0.99. Below in Figs. 6, 7 and 8 six examples of time evolution for these solutions obtained in numerics are presented. Profiles of solutions at time instants $t=0, T / 4, T / 2,3 T / 4$ and $T$, where $T=\lambda / v$ are displayed. Open symbols represent the profiles at $t=T$ which overlap with those at $t=0$ with numerical deviations less than $10^{-11}$. In all the presented examples, as well as all others not shown here, numerics confirmed a uniform motion and a fixed shape for the considered solutions (Figs. 8, 9, 10).

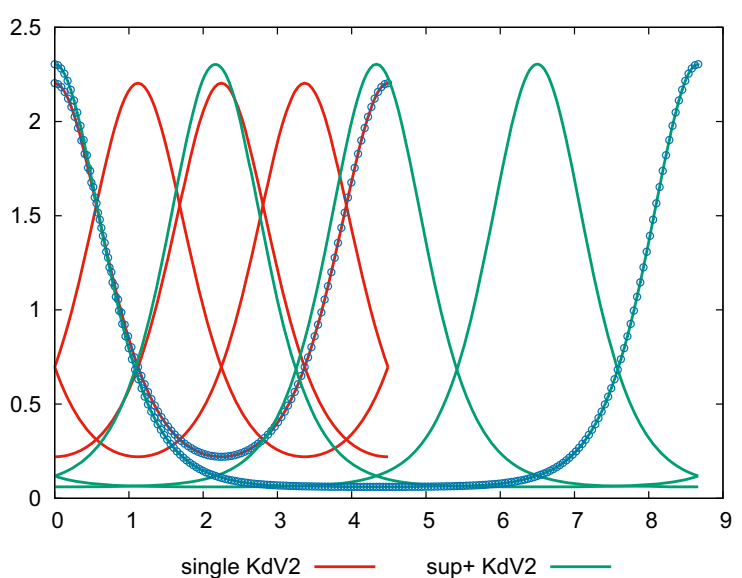

Fig. 9 Same as in Fig. 8 but for $m=0.9$. (Color figure online)

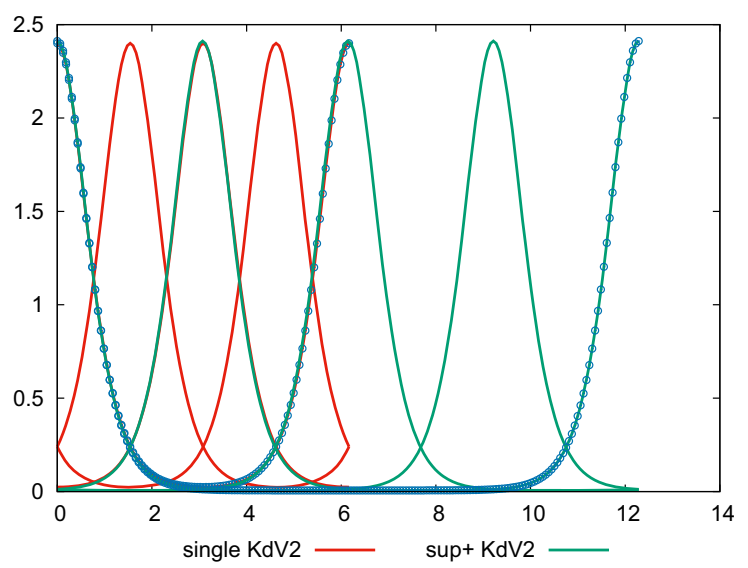

Fig. 10 Same as in Fig. 8 but for $m=0.99$. (Color figure online)

\section{Conclusions}

The most important results of the paper can be summarized as follows.

It is shown that several kinds of analytic solutions of $\mathrm{KdV} 2$ have the same forms as corresponding solutions to $\mathrm{KdV}$ but with different coefficients. This statement is true for our single solitonic solutions [10], periodic solutions in the form of single Jacobi elliptic functions $\mathrm{cn}^{2}[18]$ or $\mathrm{dn}^{2}$, and for periodic solutions in the form of superpositions $\mathrm{dn}^{2} \pm \sqrt{m} \mathrm{cn} d \mathrm{n}$ (this paper). Coefficients $A, B, v$ of these solutions to $\mathrm{KdV} 2$ are fixed by coefficients of the equation, that is by values of $\alpha, \beta$ parameters. This is in contradiction to the $\mathrm{KdV}$ case where one coefficient (usually $A$ ) is arbitrary. 
Open Access This article is distributed under the terms of the Creative Commons Attribution 4.0 International License (http://creativecommons.org/licenses/by/4.0/), which permits unrestricted use, distribution, and reproduction in any medium, provided you give appropriate credit to the original author(s) and the source, provide a link to the Creative Commons license, and indicate if changes were made.

\section{References}

1. Stokes, G.G.: On the theory of oscillatory waves. Camb. Trans. 8, 441-473 (1847). (Papers 1, 197-229.)

2. Korteweg, D.J., de Vries, G.: On the change of form of the long waves advancing in a rectangular canal, and on a new type of stationary waves. Philos. Mag. (5) 39, 422 (1985)

3. Benjamin, T.B., Bona, J.L., Mahony, J.J.: Model equations for long waves in nonlinear dispersive media. Philos. Trans. R. Soc. Lond. A 272, 47-78 (1972)

4. Bona, J.L., Pritchard, W.G., Scott, L.R.: An evaluation of a model equation for water waves. Philos. Trans. R. Soc. Lond. A 302, 457-510 (1981)

5. Infeld, E., Rowlands, G., Senatorski, A.: Instabilities and oscillations in one and two dimensional KadomtsevPetviashvili waves and solitons. Proc. R. Soc. Lond. A 455, 4363-4381 (1999)

6. Marchant, T.R., Smyth, N.F.: The extended Korteweg-de Vries equation and the resonant flow of a fluid over topography. J. Fluid Mech. 221, 263-288 (1990)

7. Marchant, T.R., Smyth, N.F.: Soliton interaction for the Korteweg-de Vries equation. IMA J. Appl. Math. 56, 157-176 (1996)

8. Burde, G.I., Sergyeyev, A.: Ordering of two small parameters in the shallow water wave problem. J. Phys. A Math. Theor. 46, 075501 (2013)

9. Karczewska, A., Rozmej, P., Rutkowski, Ł.: A new nonlinear equation in the shallow water wave problem. Phys. Scr. 89, 054026 (2014)
10. Karczewska, A., Rozmej, P., Infeld, E.: Shallow-water soliton dynamics beyond the Korteweg-de Vries equation. Phys. Rev. E 90, 0129076 (2014)

11. Karczewska, A., Rozmej, P., Infeld, E.: Energy invariant for shallow water waves and the Korteweg-de Vries equation: doubts about the invariance of energy. Phys. Rev. E 92, 053202 (2015)

12. Ali, A., Kalisch, H.: On the formulation of mass, momentum and energy conservation in the KdV equation. Acta Appl. Math. 133, 113-131 (2014)

13. Sergyeyev, A., Vitolo, R.: Symmetries and conservation laws for the Karczewska-Rozmej-Rutkowski-Infeld equation. Nonlinear Anal. Real World Appl. 32, 1-9 (2016)

14. Karczewska, A., Rozmej, P., Infeld, E., Rowlands, G.: Adiabatic invariants of the extended KdV equation. Phys. Lett. A 381, 270-275 (2017). arXiv:1512.01194

15. Khare, A., Saxena, A.: Linear superposition for a class of nonlinear equations. Phys. Lett. A 377, 2761-2765 (2013)

16. Khare, A., Saxena, A.: Superposition of elliptic functions as solutions for a large number of nonlinear equations. J. Math. Phys. 55, 032701 (2014)

17. Khare, A., Saxena, A.: Periodic and hyperbolic soliton solutions of a number of nonlocal nonlinear equations. J. Math. Phys. 56, 032104 (2015)

18. Infeld, E., Karczewska, A., Rowlands, G., Rozmej, P.: Solutions to the extended $\mathrm{KdV}$ equation for water surface waves. arXiv: 1612.03847

19. Infeld, E., Karczewska, A., Rowlands, G., Rozmej, P.: Possible existence of a new class of shallow water waves. (2017) Submitted 